\title{
Comparação de algoritmos de escolha automática de tempo de chegada de onda $\mathbf{P}$
}

Gabriel R. S. Costa (Faculdade de Geofísica-UFPA), José J. S. de Figueiredo (Faculdade de Geofísica-UFPA e INCT-GP), João Rafael Silveira(Faculdade de Geofísica-UFPA), Marcus Vinicius Sousa Corrêa (Faculdade de Geofísica) e Daniel Leal Macedo (Faculdade de Geofísica-UFPA e INCT-GP)

Copyright 2018, SBGf - Sociedade Brasileira de Geofísica

Este texto foi preparado para a apresentação no VIII Simpósio Brasileiro de Geofísica, Salinópolis, 18 a 20 de setembro de 2018. Seu conteúdo foi revisado pelo Comitê Salinópolis, 18 a 20 de setembro de 2018. Seu conteúdo foi revisado pelo Comitê
Técnico do VIII SimBGt, mas não necessariamente representa a opinião da SBGf ou Técnico do VIII SimBGf, mas não necessariamente representa a opinião da SBGf ou propósitos comerciais sem prévia autorização da SBGf.

\section{Resumo}

Este trabalho visa comparar dois métodos de escolha automática do tempo de chegada de onda $P$ em formas de ondas oriundas de amostras sintéticas (isotrópicas e anisotrópicas) em ambiente ultrassônico. $O$ primeiro método se baseia em uma modificação da razão STA/LTA, a qual é utilizada tanto na sísmica quanto na sismologia. $O$ outro método utiliza uma deconvolução tipo spiking que relaciona o sinal sísmico com a assinatura da fonte conhecida. Após a comparação destas duas formas de se obter o tempo de primeira chegada da onda $P$, percebemos que a razão STA/LTA modificada mostrou-se mais eficiente que o método baseado na deconvolução. Além de mais eficiente em seus resultados, apresenta vantagem no uso por seu algoritmo ser mais simples em comparação ao que foi utilizado na deconvolução.

\section{Introdução}

A determinação do tempo de chegada de ondas $P$ e $S$ são de fundamental importância para o processamento sísmico. Este tipo de aquisição pode ser feita manualmente, porém, acarreta em diversas desvantagens, seja por erros humanos, ou pela quantidade de ruídos que podem contaminar o sinal, dificultando a visualização. Sabendo disto, vários algoritmos para obter estas chegadas foram feitos. Estes métodos variam em grau de complexidade, precisão e aplicações. Um dos métodos mais utilizados é a razão STA/LTA (Allen, 1978) que realiza medias de amplitude em janelamentos de tempo distintos, a fim de obter o tempo de chegada da onda $P$. Outros métodos mais complexos e avançados já foram criados, como, por exemplo, Tong e Kennett (1996) usaram amplitudes simples e limiares de energia, já Withers et al. (1998) compararam sistematicamente vários algoritmos de trigger. Takanami e Kitagawa (2003) usaram abordagem de modelo auto regressiva dupla e transformada wavelet para detectar onda $P$ e onda $S$, respectivamente. Contudo, o método STA/LTA continua tendo preferência, devido a simplicidade de programar e facilidade na leitura dos resultados.

Uma grande desvantagem da razão STA/LTA é a exigência de uma razão sinal/ruído muito alta, o que normalmente é difícil de ser obtido. Por isto, foi feita uma modificação no método, de modo que a razão sinal/ruído não precise ser tão alta. Ao invés de utilizar a derivada da razão STA/LTA, utiliza-se a diferença entre os picos desta razão para a amostra e para a assinatura da fonte, (conhecida em amostras sintéticas). Tal método foi comparado com método baseado na deconvolução tipo spiking. Este ultimo método utiliza deconvolução do sinal da amostra em relação ao sinal da fonte. Este modo de aquisição tem um encargo computacional mais complexo e, na comparação entre os dois algoritmos não foram tão eficaz quanto o método baseado na razão STA/LTA.

\section{Desenvolvimento teórico}

\section{- Razão STA/LTA}

Para indicar o tempo de chegada da onda $P$, a razão STA/LTA calcula a amplitude média do sinal sísmico em dois janelamentos de tempo diferentes, um janelamento curto (STA), e um longo (LTA). Matematicamente este método é baseado nas equações abaixo:

$$
\begin{array}{rlr}
S T A & =\frac{1}{n s} * \sum_{j=i-n s}\left(x_{j}\right)^{2} & \\
L T A & =\frac{1}{n l} * \sum_{j=i-n l}\left(x_{j}\right)^{2} & \\
& \text { Eq (1) }
\end{array}
$$

onde ns e nl são, respectivamente, as janelas de tempo curto e longo e $\chi_{j}$ representa o sinal sísmico.

Como representado na Figura, 1, a janela de tempo curta(NS) é sensível à mudanças abruptas do sinal sísmico. Já o janelamento longo(NL) é mais sensível aos ruídos contidos no sinal. 


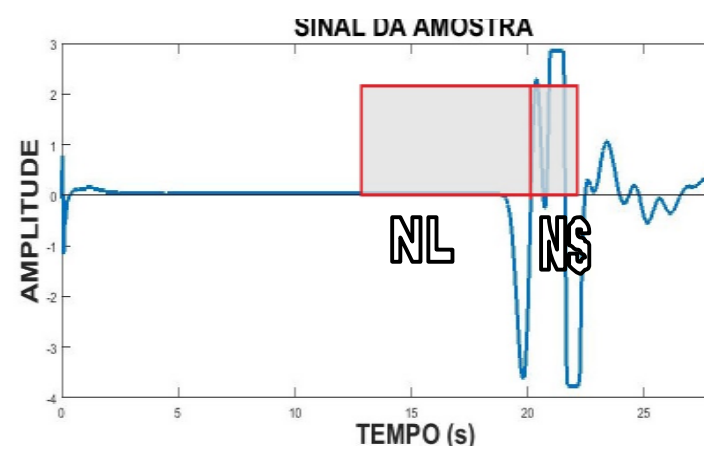

Figura 1- Representação gráfica dos janelamentos longo(NL) e curto(NL).

\section{- Deconvolução na frequência}

O outro método utilizado neste artigo tem como objetivo obter o tempo de chegada da onda $\mathrm{P}$ a partir de uma deconvolução. A partir da equação da onda escalar, é possível relacionar a reposta de uma fonte pontual por uma convolução. Esta convolução relaciona a assinatura da fonte conhecida com o traço sísmico, como mostra as equações abaixo:

$$
\begin{aligned}
\frac{\partial^{2} \varphi(x, t)}{\partial t^{2}}=c^{2} \nabla^{2} \varphi(\mathrm{x}, t)+F(\mathrm{x}, t) & , \quad \mathrm{Eq}(5) \\
\varphi(\mathrm{x}, t)=G\left(\mathrm{x}, t ; \mathbf{x}_{\mathrm{s}}, 0\right) * A(t) & \quad
\end{aligned}
$$

A equação (5) representa a equação de a onda escalar com o termo de fonte. $F(x, t)$ é o termo de fonte, enquanto c é a velocidade de propagação da onda. Já na equação 6 vemos a relação entre a assinatura da fonte, dada por $A(t)$ com o traço sísmico $G$.

A partir desta relação, será utilizado o filtro de Wiener, onde, realizando a convolução deste filtro com a wavelet(assinatura da fonte), é obtido o sinal:

$$
Y(t)=X(t) * H(t)
$$

esta relação é convertida para o domínio da frequência a partir da transformada de Fourier. O método consiste em resolver $\mathrm{H}(\mathrm{w})$ multiplicando $\mathrm{Y}(\mathrm{w})$ por um filtro $1 / \mathrm{X}(\mathrm{w})$ apropriado, $\mathrm{H}(\mathrm{w})$ neste método, é a função impulsiva que definirá o tempo de chegada da onda $P$. Além disto, é adicionado a esta relação um fator de estilização $(\epsilon)$. Tal fator tem como objetivo controlar o equilíbrio entre o grau de redução do ruído e o erro introduzido pelo filtro.

$$
H(w)=Y(w) * \frac{X^{*}(w)}{\left\{|X(w)|^{2}+\epsilon\right\}} .
$$

Figura 2 mostra exemplo do resultado de deconvolução tipo spiking. O ponto de escolha automática poderia ser atribuído ao máximo valor da saída da deconvolução.

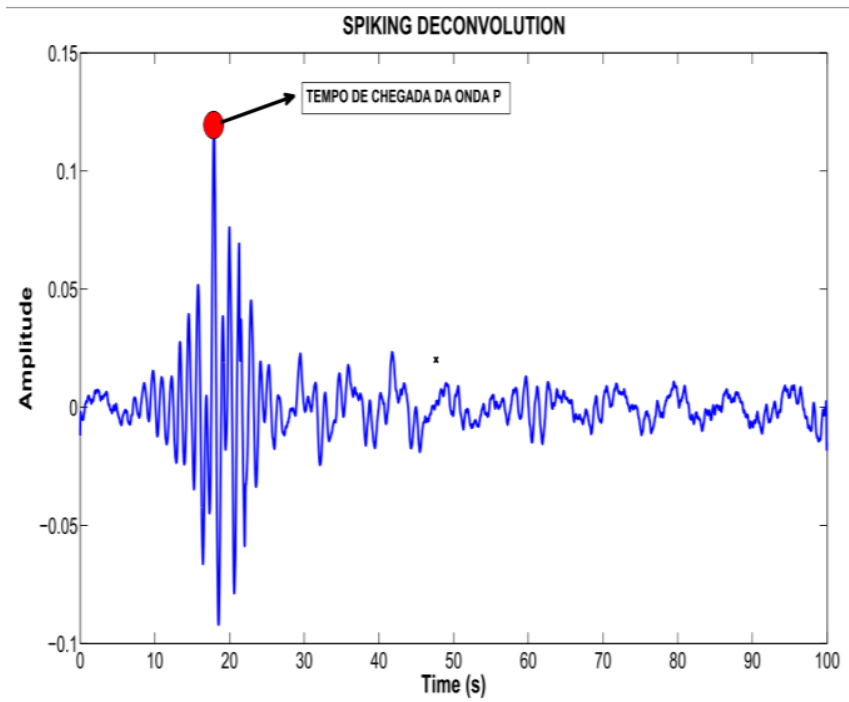

Figura 2: Representação gráfica da deconvolução entre o sinal de uma amostra isotrópica e a wavelet. O tempo de chegada da onda $\mathrm{P}$ é obtido a partir do eixo $\mathrm{x}$ do ponto máximo do gráfico.

\section{- Parâmetro de Thomsen da onda P.}

Neste trabalho, para identificar a direção do plano de fratura, é necessário calcular o parâmetro de Thomsen da onda $P(\varepsilon)$. Este valor é dado seguindo a relação abaixo:

$$
\varepsilon=\frac{V_{p}\left(90^{\circ}\right)-V_{p}\left(0^{\circ}\right)}{2 V_{p}\left(0^{\circ}\right)} \quad, \quad \mathrm{Eq}(9)
$$

onde $\mathrm{Vp}\left(90^{\circ}\right)$ é a velocidade em 90 graus e $\mathrm{Vp}\left(0^{\circ}\right)$ é a velocidade em 0 graus.

Para um caso onde a distância entre os transdutores seja igual, tanto em 90 quanto em 0 graus, a equação 9 pode ser posta em função dos tempos de transito, conforme a equação 10: 


$$
\varepsilon=\frac{T\left(90^{\circ}\right)-T\left(0^{\circ}\right)}{2 T\left(0^{\circ}\right)} \quad, \quad \mathrm{Eq}(10)
$$

onde $\mathrm{T}\left(90^{\circ}\right)$ é o tempo de transito na direção de 90 graus e $\mathrm{T}\left(0^{\circ}\right)$ é o tempo de transito em 0 graus.

\section{Metodologia:}

\section{- Comparação dos resultados obtidos em amostra isotrópica:}

Para comparar os dois métodos, foram utilizados dois tipos de amostra. Uma amostra isotrópica (veja Figura 3) e outra amostra com configuração VTI.

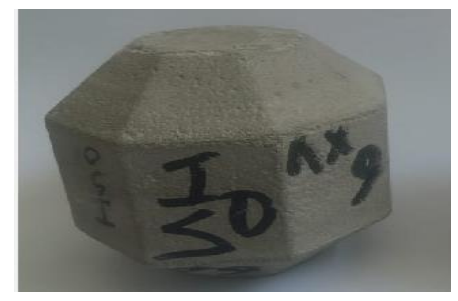

Figura 3: Amostra utilizada para comparação dos métodos.

O formato das amostras facilita as medidas nas direções $\mathrm{x}$, y e z. Além disto, o contato com os transdutores é melhor do que em formatos cilíndricos, por exemplo, o que diminui erro de contato. Na primeira comparação, utilizaremos o sinal obtido pela passagem do transdutor nos eixos $\mathrm{X}, \mathrm{Y}$ e $\mathrm{Z}$ tanto para amostra isotrópica quanto para a amostra VTI.

\section{Determinação do plano de fratura de uma amostra cilíndrica:}

Outra comparação que será feita será feita em uma amostra cilíndrica, conforme descrita na Figura 4. Nesta amostra foram realizadas medidas entre $0^{\circ}$ e $180^{\circ}$, em intervalos de 10 em 10 graus.

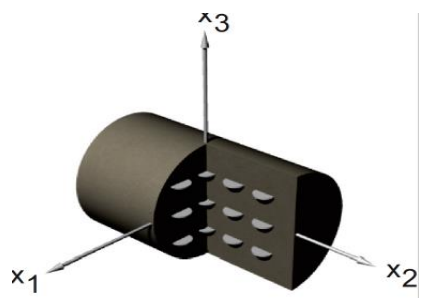

Figura 4: Representação da amostra cilíndrica na qual foi realizada a determinação do plano de fratura.

As medidas ultrassônicas foram realizadas utilizando o Sistema Ultrassônico do LPRF com a técnica de transmissão de pulso Santos et al. (2016). A taxa de amostragem por canal para todas as medidas das formas de onda $P$ foi 0,01 microssegundos. A Figura 5 mostra uma imagem deste sistema ultrassônico.

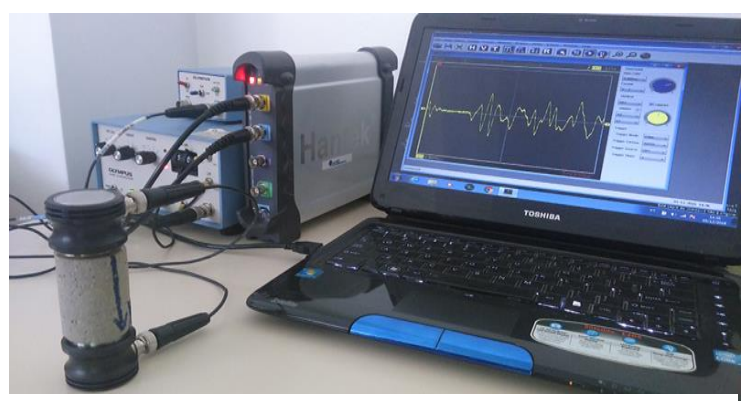

Figura 5: Sistema de aquisição do sinal das amostras

O sistema é formado por um receptor de pulso 5072PR e um pré-amplificador 5660B da Olympus, um osciloscópio USB de $50 \mathrm{MHz}$ da Handscoope, e dois transdutores de $1 \mathrm{MHZ}$ (onda P) e $500 \mathrm{kHz}$ (onda S) também da Olympus. A Figura 5 também mostra os transdutores de onda $\mathrm{P}$ e S. Os transdutores (fonte e receptor) foram dispostos em lados opostos das amostras, separados pelo comprimento da amostra. Para assegurar que a propagação da onda estava na região desejada das amostras, os transdutores foram colocados no centro da qualquer lado. Isto foi feito para ambos os modos de propagação de ondas.

Para o método da deconvolução, foi utilizado um fator de estabilização igual a 100 . Em relação à razão STA/LTA, não foi utilizado o tempo de chegada em termos da derivada, mas sim em relação à diferença entre o valor máximo dessa razão aplicada no sinal e aplicada também na assinatura da fonte.

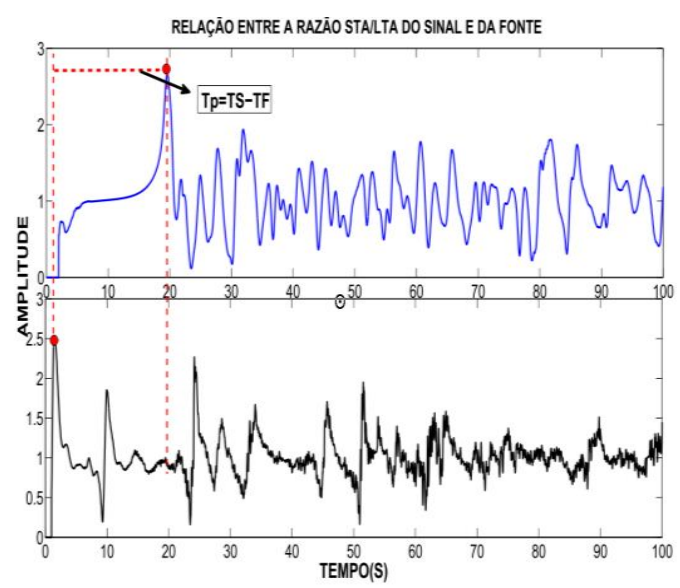

Figura 6: Modificação do método STA/LTA, reduzindo o tempo em relação aos valores máximos entre o sinal da amostra e o sinal da fonte.

Utilizar a razão STA/LTA desta maneira permite aplicar o método em sinais com uma razão sinal/ruído menor. $\mathrm{O}$ 
que é vantajoso, pois, com isso, não há perca de informação com suavizações e simplifica o algoritmo.

\section{Resultados}

Aplicando ambos os métodos para um traço obtido da amostra isotrópica no eixo $\mathrm{x}$, foram obtidos os resultados mostrados nas Figuras 7 e 8 :

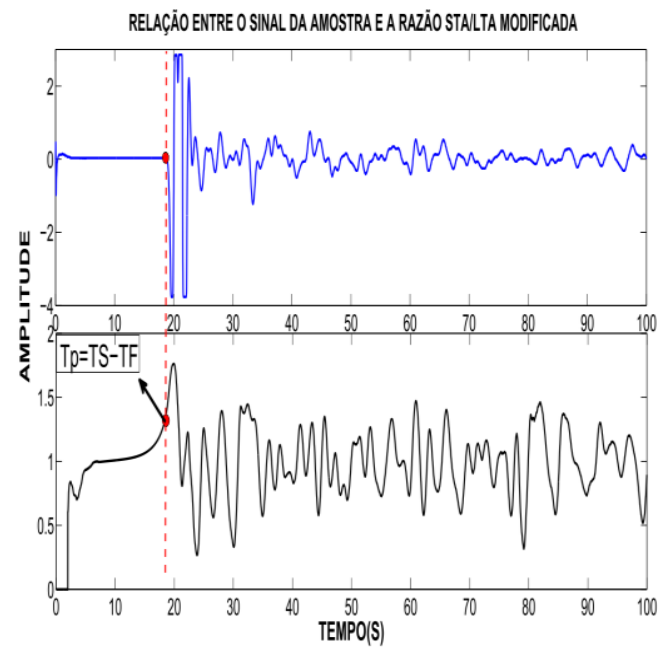

Figura 7: Relação entre a modificação do STA/LTA e o sinal sísmico.

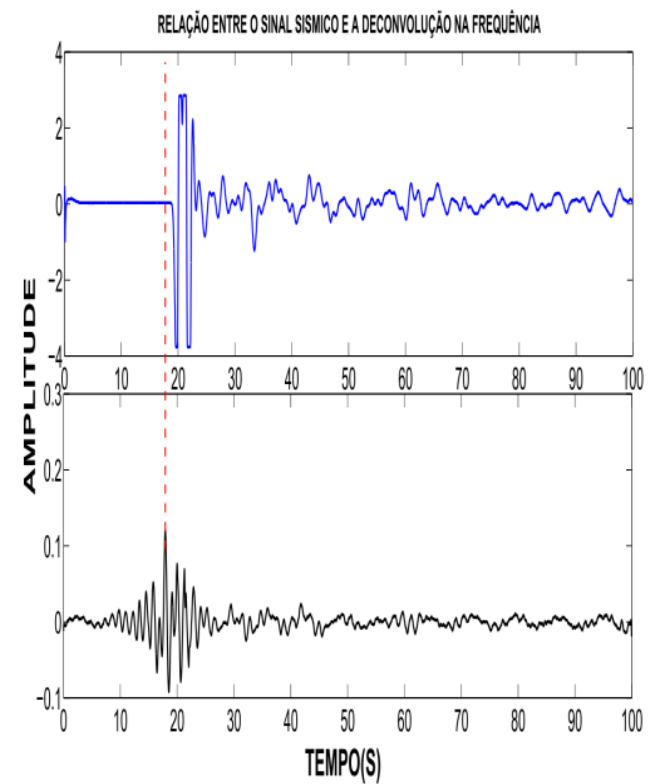

Figura 8: Relação entre a deconvolução na frequência e o sinal da amostra.

Na Figura 7, TS é o tempo de chegada do valor máximo da razão STA/LTA aplicado no sinal, enquanto TF é o tempo de chegada do valor máximo da razão STA/LTA aplicado na assinatura da fonte. Observa-se que esta subtração mostra-se satisfatória ao atingir um ponto muito próximo da máxima derivada deste sinal. $\mathrm{O}$ valor encontrado utilizando o método STA/LTA foi de 18.5 segundos, enquanto que, utilizando o método de deconvolução, o valor atingido foi de 17.91 segundos. Uma diferença de $3.2 \%$. Porém, a razão STA/LTA aproximou-se mais da onda P. As Figuras 9 e 10 mostram as medidas de tempos de chegada para as medidas que foram feitas de 10 em 10 graus. Na figura 9 estão os resultados utilizando a razão STA/LTA, enquanto a 10 mostra o resultado por deconvolução.

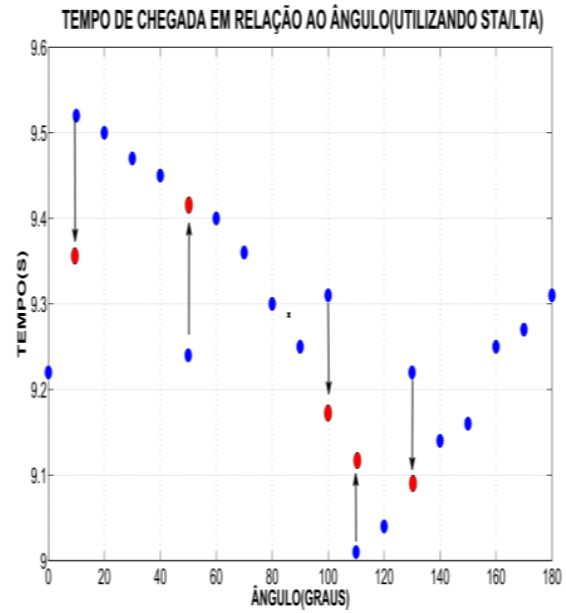

Figura 9: Medidas dos tempos de chegada em função do ângulo. O método de aquisição foi o STA/LTA.

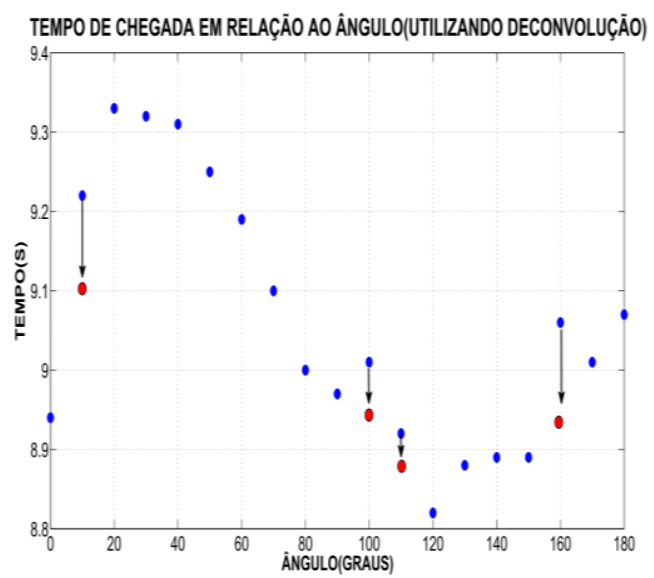

Figura 10: Medidas dos tempos de chegada em função do ângulo. O método de aquisição foi o de deconvolução no domínio da frequência

Os pontos vermelhos mostram onde os valores de algumas medidas deveriam estar em relação aos valores medidos. Utilizando-se os tempos calculados nos gráficos das Figuras 9 e 10, obtemos a direção do plano de fratura da amostra cilíndrica a partir dos dois métodos, conforme descrito na Figura 11. 

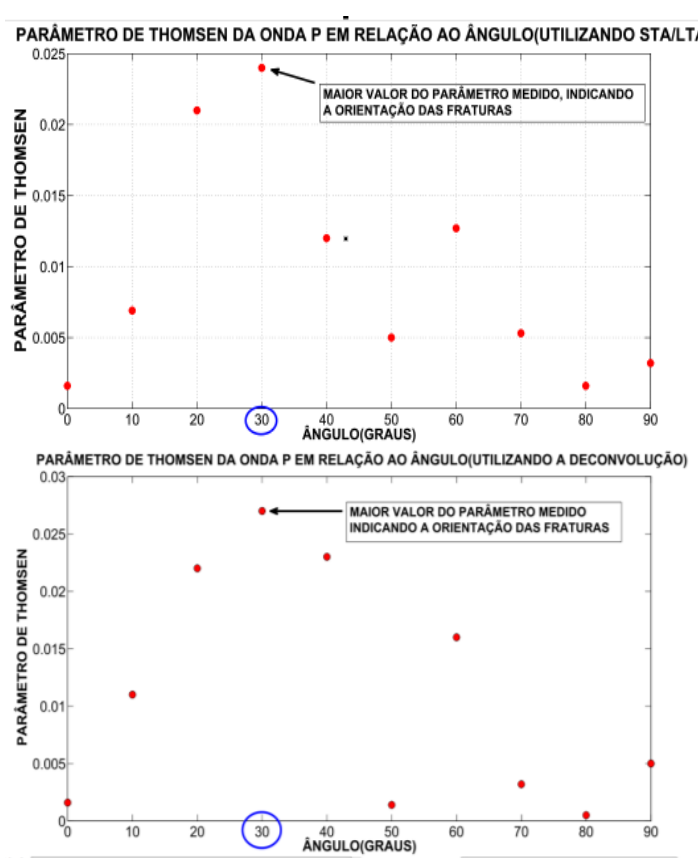

Figura 11: Representação gráfica do valor do parâmetro de Thomsen da onda $\mathrm{P}$ em relação ao ângulo para o método STA/LTA e para a deconvolução no domínio da frequência.

\section{Discussão e Conclusões}

No teste em relação à amostra isotrópica, verificamos uma diferença entre ambos os métodos em relação a pickagem feita. Constatou-se, a partir destes experimentos que o método STA/LTA utilizando os valores máximos têm uma maior eficácia e simplicidade de uso do que o método da deconvolução e o STA/LTA convencional proposto por Allen (1972).

Em relação aos erros das medidas nos ângulos de 10, 100 e 110 graus. Atribuímos os mesmos à problemas na hora de realizar a aquisição, pois, pelo formato da amostra ser cilíndrico, há uma dificuldade maior em realizar as medidas corretamente, este problema é ilustrado na Figura 12.

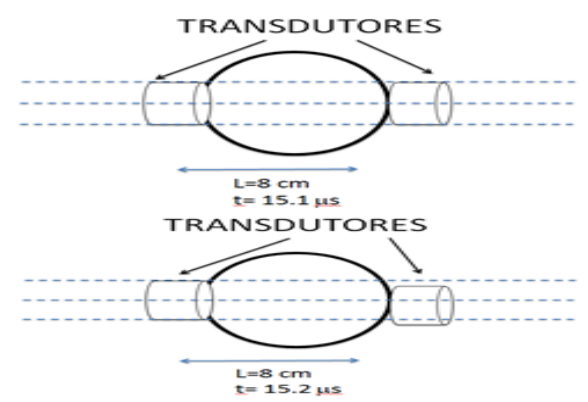

Figura 12: Representação do acoplamento dos transdutores em amostras cilíndricas. Na figura observase que qualquer desvio do transdutor pode gerar diferenças nos resultados.
Em relação ao ângulo, ambos os métodos conseguiram encontrar o valor de 30 graus para a orientação do plano de fratura, porém a diferença do valor do parâmetro de Thomsen encontrado pelos dois métodos pode dificultar outras analises que dependam deste valor.

É de extrema importância ter precisão para medir o tempo de chegada de ondas $\mathrm{P}$ e $\mathrm{S}$, além de velocidade e simplicidade. Logo, verificamos que a pickagem automática é eficiente e ajuda o profissional ou estudante a obter resultados em menos tempo e com um risco de erro menor. Verifica-se também que, alguns métodos podem ser mais eficientes que outros em determinadas situações. Em ambiente ultrassônico, a razão STA/LTA modificada apresenta melhores resultados do que 0 método da deconvolução, devido às diferenças dos resultados na amostra isotrópica e nas diferenças de valor do parâmetro de Thomsen calculado para determinar a orientação das fraturas.

Há erros embutidos em métodos automáticos de aquisição de tempo de chegada de ondas, porém, os mesmos são mais facilmente identificados, e, sobretudo, são menores do que utilizando a pickagem manual. Que exige grande experiência de quem a realizar e, muitas vezes, pode ser uma atividade cansativa e tediante.

\section{Referências}

Wong, J., Han, and L., Stewart, R.R., and Bancroft, J.C., 2009, Geophysical well logs from a shallow test well and automatic time-picking on full-waveform sonic logs: CSEG Recorder, 34, no. 4, 20-29.

Wong, J., Han L,Stewart, R.R, Bancroft, J.C,2009, Automatic time-picking of first arrivals on noisy micro seismic data, CSEG

HELBIG, K. Seismic anisotropy: Handbook of geophysical exploration, unpublished manuscript. 1994. DIX, C. H. Seismic velocities from surface measurements. Geophysics, v.20, n.01, p.68-86, 1955

Takanami, Tetsuo \& Kitagawa, Genshiro. (1988). A new efficient procedure for estimation of onset times of seismic waves. Journal of Physics of the Earth. 36. 267290. 10.4294/jpe1952.36.267.

De Figueiredo, J.J.S., J .Schleicher, R.R. Stewart, and N.Dyaur,2012, Estimating fracture orientation from elastic-wave propagation: An ultrasonic experimental approach: Journal of geophysical research: Solid Earth,117, N08304.

Santos,L.K., J.J. S. de Figueiredo, C.B. Silva, D.L Macedo, and A.M.Leandro,2017. A new way to construct synthetic porous fractured medium: journal of petroleum Science and Eng., Accepeted.

Allen, R.V., 1978. Automatic earthquake recognition and timing from single traces, Bull. seism. Soc. Am., 68, 1521-1532. 